\title{
Boundary conditions for free A-DNA in solution and the relation of local to global DNA structures at reduced water activity
}

\author{
Dietmar Porschke ${ }^{1}$
}

Received: 12 October 2015 / Revised: 30 November 2015 / Accepted: 17 December 2015 / Published online: 13 February 2016

(C) The Author(s) 2016. This article is published with open access at Springerlink.com

\begin{abstract}
Because of repeated claims that A-DNA cannot exist without aggregation or condensation, the state of DNA restriction fragments with $84-859$ bp has been analyzed in aqueous solutions upon reduction of the water activity. Rotational diffusion times $\tau^{\mathrm{d}}$ measured by electric dichroism at different water activities with a wide variation of viscosities are normalized to values $\tau^{\mathrm{c}}$ at the viscosity of water, which indicate DNA structures at a high sensitivity. For short helices (chain lengths $\ell \leq$ persistence length $p$ ), cooperative formation of A-DNA is reflected by the expected reduction of the hydrodynamic length; the transition to the A-form is without aggregation or condensation upon addition of ethanol at monovalent salt $\leq 1 \mathrm{mM}$. The aggregation boundary, indicated by a strong increase of $\tau^{\mathrm{c}}$, is shifted to higher monovalent salt $(\geq 4 \mathrm{mM})$ when ethanol is replaced by trifluoroethanol. The BA transition is not indicated anymore by a cooperative change of $\tau^{\mathrm{c}}$ for $\ell \gg p ; \tau^{\mathrm{c}}$ values for these long chains decrease upon reduction of the water activity continuously over the full range, including the $\mathrm{BA}$ transition interval. This suggests a non-cooperative BC transition, which induces DNA curvature. The resulting wide distribution of global structures hides changes of local length during the BA transition. Free A-DNA without aggregation/condensation is found at low-salt concentrations where aggregation is inhibited and/ or very slow. In an intermediate range of solvent conditions, where the A-form starts to aggregate, a time window remains that can be used for analysis of free A-DNA in a quasi-equilibrium state.
\end{abstract}

Dietmar Porschke

dpoersc@gwdg.de

1 Max Planck Institut für biophysikalische Chemie, 37077 Göttingen, Germany
Keywords Electric dichroism - Rotational diffusion · Worm-like chain - Rise per base pair - DNA state diagram . Condensation

\section{Introduction}

The transition of B- to A-DNA is observed in vitro under the special conditions of strongly reduced water activity (Franklin and Gosling 1953). Partly because of these unusual conditions, A-DNA is not always accepted as an entirely natural form of DNA, although the A-form of DNA has been demonstrated to exist in the natural environment of protein-DNA complexes (Cheetham and Steitz 1999; Jacobo-Molina et al. 1993; Jones et al. 1999; Kiefer et al. 1998; Lu et al. 2000). A special role for A-DNA has been indicated recently by its detection in an extremophile (DiMaio et al. 2015), living under extreme conditions of high temperature and low $\mathrm{pH}$. In vitro, the water activity is commonly reduced for induction of A-DNA by addition of alcohol (Brahms and Mommaerts 1964). It is well known that addition of alcohol induces precipitation of DNA (Girod et al. 1973; Shapiro 1981). In fact, formation of A-DNA has often been observed together with aggregation. Thus, the conclusion appears to be obvious and has been presented by many different authors that generation of A-DNA is always coupled with aggregation or condensation (Herbeck et al. 1976; Nishimura et al. 1985; Zimmerman and Pheiffer 1979).

This view was recently presented again by Hormeno et al. (2011), based on single-molecule experiments using force-extension with optical tweezers. Hormeno et al. claimed that "condensation prevails over B-A transition in the structure of DNA at low humidity" and that "no contour length change compatible with a cooperative transition 
between the A and B forms" was found. The question of an inherent coupling between the BA transition and aggregation or condensation is not only of academic but also of practical interest. Such coupling is essential for the interpretation of both equilibrium and kinetic data.

Most of the available data on the BA transition have been collected by Ivanov et al. (e.g. Ivanov and Krylov 1992; Ivanov et al. 1974) at low-salt concentrations based on experimental evidence that aggregation is avoided at lowsalt conditions. It is remarkable that the authors claiming a crucial role of aggregation for the BA transition usually do not consider the arguments of the authors supporting the existence of free A-DNA. Are these arguments not convincing enough? Basic physical chemistry tells that aggregation of highly charged objects is suppressed at low-salt conditions, but experimental details should be examined carefully. Aggregation is dependent on many different parameters and experimental techniques for unequivocal exclusion and/or quantification of aggregation are usually elaborate and require experience. This seems be the reason why these techniques have been applied only to a limited extent.

Analytical ultracentrifugation was used by Herbeck et al. (1976) for calf thymus DNA at $0.2 \mathrm{mM}$ salt: at $70 \%$ $\mathrm{EtOH}$, the sedimentation coefficient was identical to that in water, whereas at $80 \% \mathrm{EtOH}$ a strong increase indicated aggregation. The authors suggest that "aggregation stabilizes the A form through lateral interactions between the helices." In addition, they comment that "it seems to be apparent that DNA cannot even exist in the A-form as a single isolated chain in solution". Potaman et al. (1980) compared in a subsequent analysis of calf thymus and T7 phage DNA at $0.5 \mathrm{mM} \mathrm{NaCl}$ the changes of the sedimentation coefficients and the $\mathrm{CD}$ spectra upon addition of $\mathrm{EtOH}$ in the BA transition range. They found aggregation of DNA at high EtOH percentage. However, the change of sedimentation coefficients was still small at a degree of $\sim 66 \%$ transition to the A-form assigned by their CD data. This is the basis for their conclusion that "aggregation is not a necessary condition for the B $\rightarrow$ A transition."

Ivanov's group (1978) determined the degree of DNA orientation in a flow within the interval of the BA transition and found nearly identical orientation below and above the transition; they conclude "that there is no aggregation in the course of formation of the A form." However, some change of the flow-induced orientation is expected to result from the length reduction during the BA transition. Thus, the question is raised again: are the arguments in favor of A-DNA without aggregation at low salt convincing enough? The molecular interactions contributing to the BA transition are of sufficiently general interest for an independent careful analysis.

Ivanov et al. claimed repeatedly that aggregation can be suppressed by using trifluoroethanol instead of ethanol for reduction of the water activity. However, direct experimental data in support of this statement apparently have not been published. Because of this gap in the literature, the present investigation has been extended to the case of trifluoroethanol.

Structures in solution and potential aggregation may be analyzed at a particularly high sensitivity by measurements of rotational diffusion. Electro-optical techniques are most attractive for the analysis of DNA because the high optical anisotropy of DNA together with the high electrical anisotropy at low-salt concentrations used for A-DNA provides an optimal basis for accurate results (Fredericq and Houssier 1973; Porschke and Antosiewicz 2007). Some reports on electro-optical measurements of A-DNA have been published (Charney and Chen 1987; Wu et al. 1981), partly with contradictory results. In the present investigation, the BA transition is analyzed in more detail over a range of different chain lengths and with special emphasis on the aggregation/condensation controversy. The experimental data demonstrate the state of DNA in solution and the boundary conditions for free A-DNA at a high accuracy and reliability. Moreover, the results provide new information about the transition from B- via C- to A-DNA and explain the nature of the problem with detection of length changes during the BA transition described by Hormeno et al. (2011).

\section{Materials and methods}

DNA fragments were prepared as described previously (Porschke 1991b). All DNA fragments were blunt ended. Absolute ethanol for analysis was obtained from Merck (Darmstadt, Germany). 2,2,2-Trifluoroethanol ReagentPlus $\geq 99 \%$ was from Sigma; lot \# MKBQ9888V was used exclusively because this lot had a sufficiently low UV absorbance. The standard buffer B contained $1 \mathrm{mM} \mathrm{NaCl}$, $1 \mathrm{mM}$ Na-cacodylate $\mathrm{pH} 7.0$ and $0.2 \mathrm{mM}$ EDTA. A dilution of buffer B by a factor of 10 is denoted buffer A. Addition of 2 and $10 \mathrm{mM} \mathrm{NaCl}$ to buffer B resulted in buffers $\mathrm{C}$ and $\mathrm{D}$, respectively. Ethanol or trifluoroethanol was always added as the last component, except for small final additions of water to arrive at a well-defined total volume in small volumetric flasks. The alcohol content at the stage of mixing was controlled by weighing. The solutions had to be degassed before exposure to electric field pulses under vacuum, which induces some reduction of the alcohol content. For this reason, the exact alcohol content during the measurements was determined after completion of the field jump experiments by evaluation of the density using a DMA60/602 (Anton Paar, Graz, Austria). UV spectra of samples were measured before and after exposure to electric field pulses directly in the field jump cell in a PerkinElmer Lambda 17 spectrophotometer. Conductivities were controlled routinely. 
The absorbance of the DNA samples at $260 \mathrm{~nm}$ and $1 \mathrm{~cm}$ path length was in the range of $0.05-0.2$, corresponding to helix concentrations in the range of $5-150 \mathrm{nM}$, depending on the chain length. The measurements started usually $\sim 30 \mathrm{~min}$ after mixing. At high values of the alcohol content (given in volume $\% \equiv$ 'Vol\%EtOH' or 'Vol\%TFE'), a dependence of the dichroism curves on the time after mixing was observed. This effect appeared in the range, where DNA association was indicated by other criteria like turbidity as well.

The electric dichroism was measured as described previously (Grünhagen 1974; Porschke 1980). A novel construction of optical windows was used in the cells for these measurements (publication in preparation). Dichroism transients were routinely recorded at $248 \mathrm{~nm}$ for parallel orientation of the polarized light with respect to the field vector. Control experiments at the magic angle at maximal electric field strengths demonstrated the absence of field-induced denaturation. The reduced linear dichroism is given as $\xi=1.5 * \Delta E_{||} / \bar{E}$, where $\Delta E_{||}$is the stationary change of the absorbance at parallel orientation of polarized light with respect to the field vector and $\bar{E}$ is the isotropic absorbance. The temperature used for the measurements was $20 \pm 0.1{ }^{\circ} \mathrm{C}$. Time constants were fitted to dichroism decay curves by standard programs (Diekmann et al. 1982; Porschke and Jung 1985) developed previously. The time constants were always measured over a range of different electric field strengths. As described previously (Porschke 1986), there is usually an increase of these time constants with increasing field strength. Linear regression of these data provided time constants at zero electric field strength.

Because the viscosity of water alcohol mixtures shows a strong dependence on the alcohol content, the time constants had to be converted to a standard viscosity for comparison. Thus, all time constants given in figures or elsewhere are converted to the viscosity of water at $20{ }^{\circ} \mathrm{C}$ by the standard viscosity conversion factor. This correction is indicated by the superscript "c", e.g., $\tau^{c}$. Densities and viscosities for water-ethanol mixtures were taken from the CRC Handbook of Chemistry and Physics. Densities and viscosities for water-trifluoroethanol (TFE) mixtures were obtained from Esteve et al. (1995) and Olive et al. (1996). Polynomial fitting and interpolation was used for evaluation of vol\% EtOH or vol\% TFE from measured densities and for determination of viscosity values at given vol\% EtOH or vol\% TFE.

\section{Results}

\section{Rotational diffusion time constants}

DNA fragments with chain lengths in the range up to $\sim 100 \mathrm{bp}$ are below the persistence length and thus can be considered as rigid rods at a reasonable approximation. In

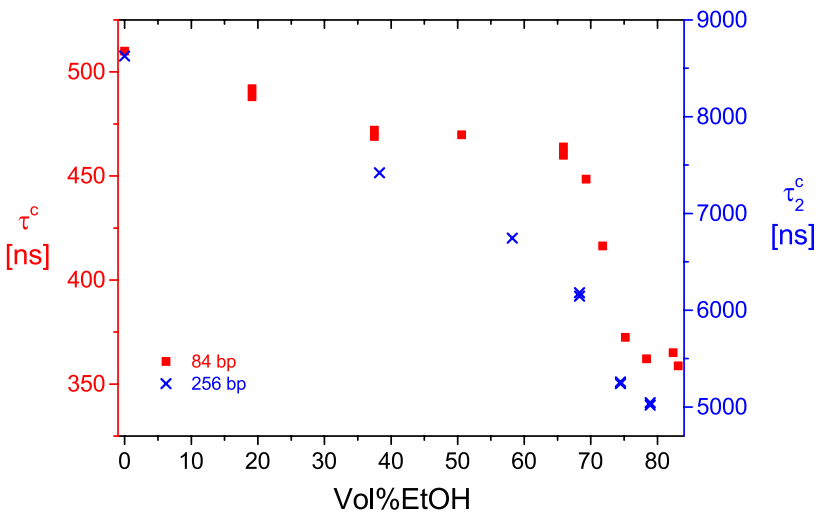

Fig. 1 Dichroism decay time constants of 84- and 256-bp DNA fragments at $20.0{ }^{\circ} \mathrm{C}$ in buffer $\mathrm{A}$ as a function of the ethanol content in vol\%. The scale on the left side represents the single exponential time constants $\tau^{\mathrm{c}}$ for the 84-bp fragment; the scale on the right side represents the slow component $\tau_{2}^{\mathrm{c}}$ of double exponential fits for the 256-bp fragment

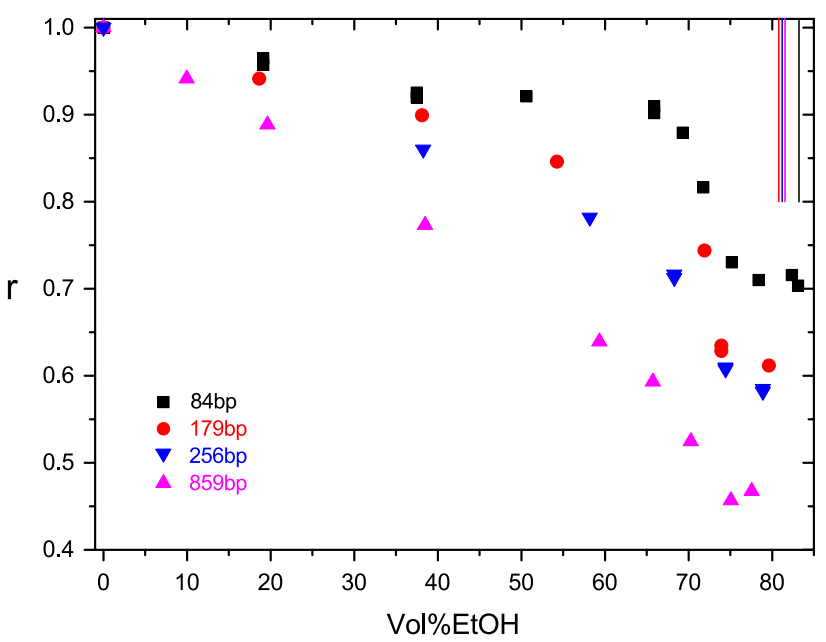

Fig. 2 Ratio $r$ of the dichroism decay time constants measured at a given ethanol content to that measured without ethanol addition at $20{ }^{\circ} \mathrm{C}$ in buffer $\mathrm{A}$ as a function of the ethanol content in vol\% for different DNA fragments. The vertical lines (color code as in the symbols) represent approximate values for the limit ethanol content, where aggregation starts to prevail

this limit, the dichroism decay can be fitted by single time constants. Measurements for a fragment with $84 \mathrm{bp}$ in buffer A as a function of the ethanol content (Fig. 1) demonstrate a strong decrease of the time constants normalized to the viscosity of water $\tau^{\mathrm{c}}$ in a narrow range of the ethanol content, indicating the BA transition. Some decrease of the time constants is observed already in the range of vol\% EtOH below the BA transition, but this effect remains relatively small. For comparison, data obtained for a fragment with 256 base pairs are shown, scaled upon the data for the shorter fragment. Fitting of the dichroism decay of the longer fragment 


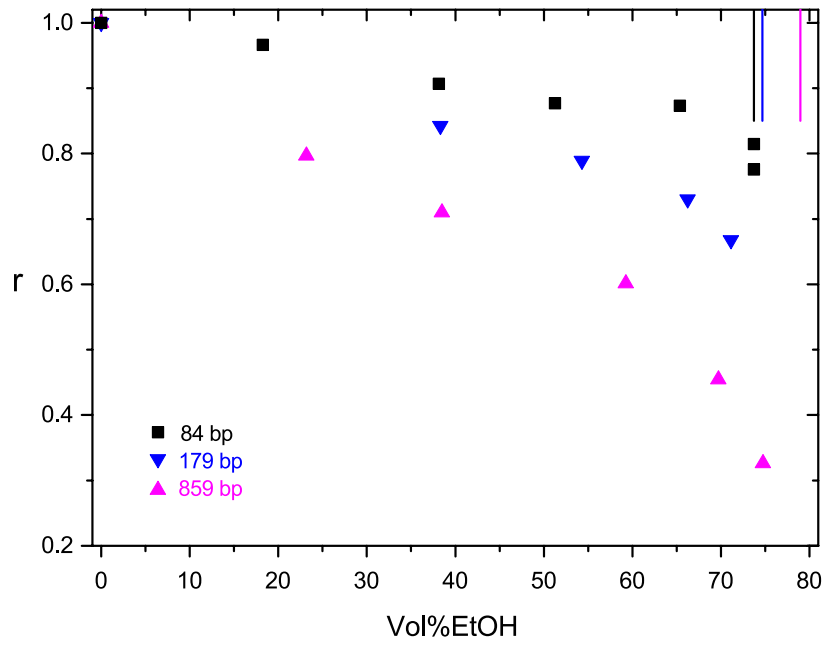

Fig. 3 Ratio $r$ of the dichroism decay time constants measured at a given ethanol content to that measured without ethanol addition at $20{ }^{\circ} \mathrm{C}$ in buffer B as a function of the ethanol content in vol\%. The vertical lines (color code as in the symbols) represent approximate values for the limit ethanol content, where aggregation starts to prevail

requires two time constants. The second time constant $\tau_{2}^{\mathrm{c}}$ reflects the overall hydrodynamic dimensions and is shown in Fig. 1. For the 256 bp fragment, there is again a cooperative decrease in the range of the BA transition, but the amplitude of this decrease is small compared to the relatively large decrease in the preceding vol\%EtOH range.

A further shift in the dependence of the time constant on the water activity is observed for the fragment with $859 \mathrm{bp}$. In this case, the BA transition is not reflected anymore by a clear cooperative decrease of the time constant in the expected narrow range of the water activity. Instead, there is an overall continuous decrease of the time constant extending from 0 to 80 vol\%EtOH. This is shown in Fig. 2 in terms of ratios of time constants measured in buffer $\mathrm{A}$ at given vol\%EtOH relative to the respective time constants found in the absence of ethanol. The dependence of the ratio $r$ on the ethanol content for different DNA fragments shows a continuous decrease of the change $\Delta r_{\mathrm{A}}$ in the BA transition range with increasing chain length, whereas the change $\Delta r_{\mathrm{C}}$ in the range below the BA transition increases. Corresponding data at a higher salt concentration (buffer B) are shown in Fig. 3. The dependence is similar to that found in buffer $\mathrm{A}$, but the limit of aggregation is shifted to lower vol\%EtOH at the higher salt concentration. Thus, the main part of the BA transition is hidden in buffer B even for the 84-bp fragment by aggregation effects.

\section{Aggregation}

It is well known that DNA can be condensed, aggregated, or precipitated by addition of ethanol (Bloomfield 1996;

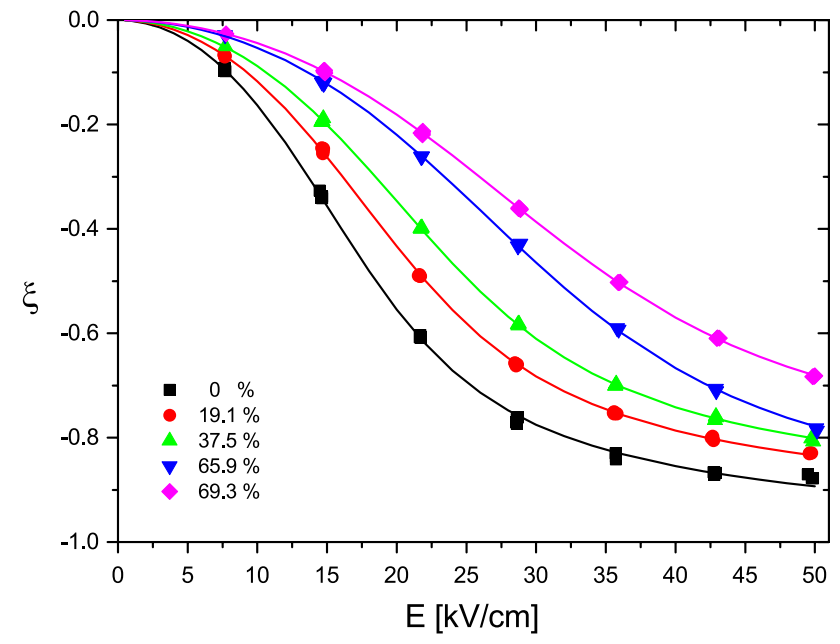

Fig. 4 Reduced linear dichroism $\xi$ for the 84-bp fragment in buffer A at $20{ }^{\circ} \mathrm{C}$ as a function of the electric field strength $\mathrm{E}$ at different ethanol contents in vol\%. The lines show least squares fits by the saturating induced dipole model; parameters are given in Fig. 5

Girod et al. 1973; Lang 1969; Shapiro 1981). Precipitation is the final stage of a process going in different reaction steps, which are affected by various parameters, including the ionic conditions, the DNA concentration, and chain length. Because the main goal in the present context is avoidance of these "perturbations", observations on aggregation effects are presented here within these limits and without a systematic investigation. Electro-optical time constants are very sensitive indicators of aggregation, particularly in the range of chain lengths $\leq$ persistence length. In the case of the 84-bp fragment in buffer A, the first indication for aggregation appeared at $75.17 \mathrm{vol} \% \mathrm{EtOH}$ in the form of a slow component with $\tau_{2}^{\mathrm{c}} \geq 20 \mu \mathrm{s}$ in the dichroism decay. The relative amplitude of this component was $1.7 \%$ in the range of $50 \mathrm{kV} / \mathrm{cm}$, where the overall degree of orientation approaches a high level (cf. Fig. 4) and thus the amplitude percentage reflects the population percentage at a sufficiently high accuracy. At lower field strengths, the slow relative amplitude is higher due to a relatively high polarizability of the aggregates. At this stage, the decay time constants $\tau_{1}^{\mathrm{c}}$ of the DNA monomers can still be measured at a high accuracy because of their large relative amplitude ( $>90 \%$ at $\sim 50 \mathrm{kV} / \mathrm{cm}$ ). When the EtOH content is increased, the relative amplitude of the slow component increases. A test experiment revealed that the slow amplitude observed in the range of vol\%EtOH $>75$ increases slowly with time, indicating a slow association reaction [cf. (Hillen and Wells 1980)].

These observations demonstrate that there is a transition range, where part of the DNA starts to be aggregated, but the free form of A-DNA can still be characterized at a high accuracy. When the EtOH content is increased further 
under the conditions of the present experiments, limit values of vol\%EtOH appear, beyond which aggregation is dominant and the characterization of free A-DNA requires a higher accuracy and more efforts. These limit values are indicated by vertical lines in Figs. 2 and 3.

In the vol\%EtOH-range with initial aggregation, measurements of UV spectra as a function of time after EtOH addition show the existence of an induction period with relatively small and slow changes of the absorbance followed by a period with relatively fast and large absorbance changes. The existence of such induction periods may be used for the characterization of A-DNA in a transient state under limit conditions.

Separate amplitudes with time constants larger by factors of $\sim 100$ than those of the "free" state are also observed at $\mathrm{vol} \% \mathrm{EtOH} \geq 80$ for the fragments with 179 and $256 \mathrm{bp}$ in buffer $\mathrm{A}$. The analysis is more difficult for still longer fragments, because the broad range of wormlike chain configurations of long chains leads to a broad range of time constants. The time constants observed for the fragment with $859 \mathrm{bp}$ in buffer A are larger in the range vol\% EtOH $\geq 80$ than those found at lower ethanol contents, but the increase is not nearly as much as observed for the shorter fragments. It is conceivable that there is intramolecular condensation of long fragments, at least partly, in contrast to the intermolecular aggregation indicated by the present results for relatively short fragments. These phenomena should be analyzed in more detail, as intra-molecular condensation effects may be distinguished from inter-molecular aggregation on the basis of their kinetics (Porschke 1984, 1991a).

When the salt concentration is increased from $0.24 \mathrm{mM}$ (buffer A) to $2.4 \mathrm{mM}$ (buffer B), aggregation effects appear at lower ethanol contents (cf. Fig. 3). Aggregation effects and the BA transition are observed in about the same range of ethanol content in buffer B for the restriction fragments used in the present investigation. Experiments on the 84-bp fragment in buffer D demonstrate a further decrease of the aggregation limit to $\sim 66 \mathrm{vol} \% \mathrm{EtOH}$ at the ionic strength of $12.4 \mathrm{mM}$.

\section{Evaluation of the rise per base pair}

The fragment with $84 \mathrm{bp}$ in buffer A shows a cooperative reduction of the hydrodynamic length upon the transition to the A-form. Because the 84-bp fragment is rather close to the limit of a rigid rod, the experimental time constants may be used for an evaluation of the rise per base pair. As demonstrated by comparison of values for different limit models (see below), DNA bending effects should be considered for an increased accuracy. The length dependence of the $\tau^{\mathrm{d}}$ values for blunt-ended restriction fragments in buffer A without ethanol indicates a persistence length of $2000 \AA \AA$ and a hydrodynamic helix radius of $12 \AA$.
The dichroism decay time constant for the 84-bp fragment found in the absence of ethanol $\tau^{\mathrm{d}}=510 \mathrm{~ns}$ decreases to $\tau^{\mathrm{c}}=362 \mathrm{~ns}$ upon formation of A-DNA (at vol $\% \mathrm{EtOH}>78$; cf. Fig. 1). The model for rigid rods by Tirado and Garcia de la Torre (1980) predicts a time constant of $532 \mathrm{~ns}$ for a rise per base pair of $3.4 \AA$ and a radius of $12 \AA$. Application of the wormlike chain correction by Hagerman and Zimm (1981) provides a value of $515 \mathrm{~ns}$ for a persistence length of $2000 \AA$. This comparison shows that the 84-bp fragment is rather close to a rigid rod, but the time constants are already affected by the flexibility/curvature to some degree. A corresponding calculation assuming the same helix radius and persistence length with a rise per base pair of $2.94 \AA$ provides the values 370 and $361 \mathrm{~ns}$ for the rigid rod and the flexible rod, respectively. Thus, the experimental $\tau^{\mathrm{c}}=362 \mathrm{~ns}$ for the 84-bp fragment at vol\%EtOH $>78$ indicates an average rise per base pair of $2.94 \AA$ for A-DNA in buffer A (estimated accuracy $\pm 0.05 \AA$ ).

The experimental time constant for the 84-bp fragment in buffer A at $65.9 \mathrm{Vol} \% \mathrm{EtOH}$, before the onset of the BA transition, is $462 \mathrm{~ns}$. Using again a helix radius of $12 \AA$ and a persistence length of $2000 \AA$, this time constant corresponds to a rise per base pair of $3.25 \AA$-an approximate experimental value for C-DNA under the given conditions.

\section{Linear dichroism and its limit value}

The field-induced orientation of DNA double helices is reflected by the reduced linear dichroism $\xi$. The magnitude of $\xi$ shows the degree of DNA alignment, which increases with the electric field strength $\mathrm{E}$. The dependence of $\xi$ on $\mathrm{E}$ is described by an orientation function (Fredericq and Houssier 1973), corresponding to a Boltzmann function, which can be used to evaluate the limiting linear dichroism $\xi_{\infty}$ at infinitely high electric field strength and the electrical parameters of the DNA. $\xi_{\infty}$ is a measure of the base pair orientation with respect to the helix axis and can be used to derive the average angle of the transition dipoles of the bases with respect to the helix axis.

The reduced linear dichroism $\xi$ as a function of the field strength $\mathrm{E}$ for the 84-bp fragment in buffer $\mathrm{A}$ is given in Fig. 4 at different vol\%EtOH. Fitting of these data by the orientation function for a saturating induced dipole (Diekmann et al. 1984) provides the following results (cf. Fig. 5): (1) the increase of the ethanol content induces a strong decrease of the polarizability; (2) the limit reduced dichroism $\xi_{\infty}$ remains constant within experimental accuracy. It is remarkable that the transition from the $\mathrm{B}$ - to the A-form is not associated with a clear change of the limit dichroism. The dichroism parameters for the other DNA fragments are similar. The present results on the limit linear dichroism of A-DNA are consistent with those of Charney and Chen (1987), but are different from those reported by $\mathrm{Wu}$ et al. (1981). The $\xi_{\infty}$ 


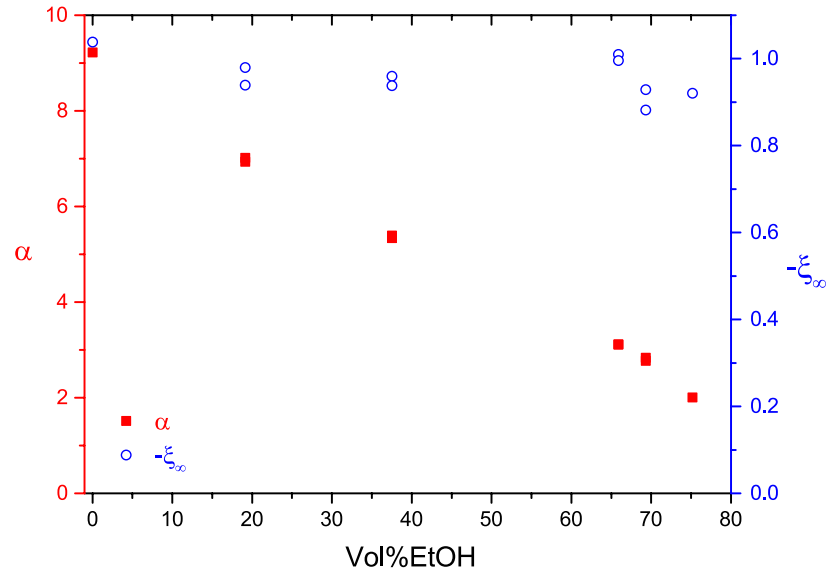

Fig. 5 Polarizability $\alpha\left(10^{-33} \mathrm{Cm}^{2} \mathrm{~V}^{-1}\right)$ and limiting value of the reduced linear dichroism $\xi_{\infty}$ for the $84 \mathrm{bp}$ fragment in buffer $\mathrm{A}$ at $20{ }^{\circ} \mathrm{C}$ as function of the ethanol content in vol\%

values indicate that the bases are inclined at an average angle of $\sim 70^{\circ}$ with respect to the helix axis both for the A- and the B-form under the given experimental conditions.

\section{Reduction of water activity by addition of trifluoroethanol}

The experimental data for DNA in water-trifluoroethanol mixtures were measured and analyzed as described for the case of ethanol. The results are very similar, but the range of experimental conditions without aggregation and precipitation is extended. Thus, free A-DNA can be observed up to higher salt concentrations. This is demonstrated for the case of the DNA fragment with $84 \mathrm{bp}$ at the $\mathrm{Na}^{+}$-concentration of $4.4 \mathrm{mM}$ in Fig. 6.

A more extensive characterization of DNA samples in various water-trifluoroethanol mixtures revealed special effects, which are not directly related to the main subject of the present investigation. These effects will be described elsewhere.

\section{Discussion}

The data obtained by electro-optical measurements provide information mainly on the global structure of DNA restriction fragments in solution. It is well known that this global structure is determined both by the flexibility of the double helix and inherent long-range structures imposed by the sequence. Separation of these effects, for example in terms of a dynamic and a static persistence length (Trifonov et al. 1987), is not simple. A very sensitive measure of the global structure is the rotational time constant, which has been determined in the present investigation from the decay of

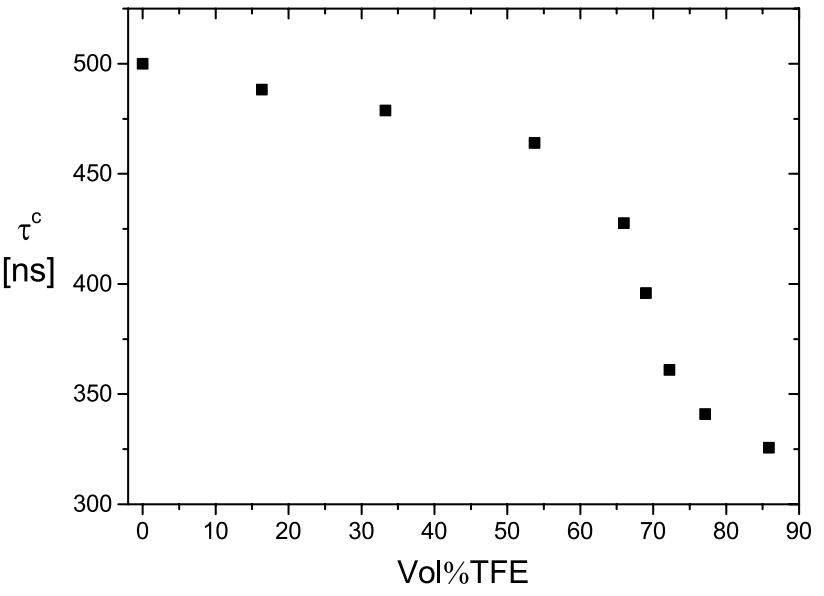

Fig. 6 Dichroism decay time constant $\tau^{\mathrm{c}}$ of the 84-bp DNA fragment at $20.0{ }^{\circ} \mathrm{C}$ in buffer $\mathrm{C}$ (ionic strength $4.4 \mathrm{mM}$ ) as a function of the trifluoroethanol content in vol\%

the field induced linear dichroism. A potential perturbation of the experimental data resulting from field-induced stretching of the global structure by the applied electric field has been avoided by extrapolation of the data to zero electric field strength.

\section{$\mathrm{B} \rightarrow \mathrm{C} \rightarrow$ A transitions}

The time constants observed for the shortest fragment with $84 \mathrm{bp}$ are particularly useful for demonstration of structure changes upon reduction of the water activity. Because the length of this fragment is below the persistence length, the interpretation of the data is relatively simple. A very clear effect is the cooperative reduction of the time constant in the range around $73 \mathrm{vol} \% \mathrm{EtOH}$, indicating the BA transition. In addition, there are two other effects that can be identified: (1) a decrease of the time constant in the vol\%EtOH range below the BA transition and (2) an increase appearing at higher vol\% EtOH than the BA transition. The latter one is expected in this regime of high vol\% EtOH and represents association of DNA strands, which finally leads to precipitation. The decrease below the BA transition has not been described yet. A comparison with observations presented in the literature indicates that it represents a BC transition. C-DNA was identified during early studies of DNA structure by fiber diffraction (Arnott and Selsing 1975; Saenger 1984; Zimmerman and Pheiffer 1980). Various experimental data show that the BC transition is non-cooperative (Brahms et al. 1973), which is consistent with the present observations. C-DNA is reported to have an axial rise per residue of $3.31 \AA$ with base pairs slightly tilted by $8^{\circ}$ and to repeat itself exactly after three turns with $28 \mathrm{bp}$. The rise per base pair obtained in the present investigation at $65.9 \mathrm{vol} \% \mathrm{EtOH}$ of $3.25 \AA$ 
is in close agreement with the literature value for C-DNA and is clearly different from the value of $3.4 \AA$ established for B-DNA (Bloomfield et al. 2000; Saenger 1984). Thus, reported properties of C-DNA including the rise per base pair and non-cooperative formation at reduced water activity agree with the present observations and the assignment seems to be sufficiently clear.

Van Dam and Levitt (2000) concluded from a combination of NMR, X-ray diffraction, and model building that $\mathrm{B}$ and $\mathrm{C}$ forms of DNA have two distinct nucleotide conformations, corresponding to the BI and BII conformations known from oligonucleotide crystals. The proportion of the BII conformation is higher in the $\mathrm{C}$ form than in the $\mathrm{B}$ form and the $\mathrm{BC}$ transition corresponds to BI-BII conformational changes of nucleotides. Tisne et al. (1998) observed helix curvature associated with BII conformations by solution NMR in a DNA hexamer. Thus, the decrease of the rotational time constants upon reduction of the water activity in a broad range of $\mathrm{vol} \% \mathrm{EtOH}$ is consistent with DNA curvature associated with a non-cooperative $\mathrm{BC}$ transition.

Because of the close similarity of B- and C-DNA, the question has been raised whether definition of a separate helix form is justified. Such doubts may continue to exist as long as a high-resolution crystal structure of C-DNA has not been found yet. Probably, C-DNA crystals have not been found because of sub-optimal crystal packing effects, e.g., resulting from curvature. Usually the assignment of structures in solution is supported by CD spectroscopy. Differences in CD spectra between B- and C-DNA have been reported. A "schematized" CD spectrum for C-DNA was presented by Ivanov et al. (1973), for example, but a different assignment was given by Bokma et al. (1987). Apparently an unequivocal deduction of a reference CD spectrum for C-DNA is difficult (Bokma et al. 1987). Under these special conditions, rotational diffusion time constants obtained from electro-optical measurements are very useful for the characterization of DNA structures in solution because of their high sensitivity. Changes of structure can be identified at a high accuracy, also those induced in solvent systems other than simple aqueous environments. It may be noted here that the solvent conditions explored in the present investigation cover a range which may be closer to that in living cells than a simple aqueous environment.

\section{Hiding of BA transition}

A special phenomenon in the set of hydrodynamic data obtained in the present investigation is the apparent disappearance of the BA transition when the chain length is increased. The hydrodynamic data demonstrate a clear decrease of the cooperative amplitude associated with the BA transition with increasing DNA chain length, whereas the amplitude appearing in the range of vol\%EtOH below the BA transition increases. The change of the time constant in the BA transition range is quite large for the fragment with $84 \mathrm{bp}$, but is hardly visible anymore at $859 \mathrm{bp}$. These data may induce the suspicion that the B-A transition is suppressed at increased chain lengths, but other experimental approaches like CD titrations (Hillen and Wells 1980), indicating the local structure, never provided any evidence for such an unusual effect. Thus, the BA transition does not disappear at high chain lengths, but is hidden in the hydrodynamic data by the $\mathrm{BC}$ transition and the wide variation of the DNA configurations at high chain lengths. This does not reduce the value of the rotational time constants, which are particularly sensitive, but illustrates the difficulty to separate different effects in the hydrodynamics of long chains. It should be emphasized that the effects described here, appearing below the aggregation limit, cannot be attributed to condensation because a total stepwise collapse is characteristic of condensation (Bloomfield 1996).

Knee et al. (2008) reported evidence for a "sequential mechanism for the A-to-B transition in DNA". They used ultraviolet resonance Raman spectroscopy, suggesting that the "transition involves a series of intermediate forms between A and B. Cooperative and distinct transitions were observed for the bases and the sugars." The present hydrodynamic data do not show evidence for such "cooperative distinct transitions" in the intermediate range of the water activity; cooperativity is found only in the standard range of the BA transition. The preceding change assigned to a $\mathrm{BC}$ transition is extended over a broad range of water activity and is without indication for cooperativity, in agreement with early observations (Brahms et al. 1973).

\section{Condensation and aggregation}

Both the BA and BC transition induced by addition of ethanol can be studied in solution only at low-salt concentrations because an increase of the salt induces condensation and aggregation. Condensation and aggregation are associated with particularly large changes of electro-optical effects and, thus, can be easily distinguished from other phenomena. The data obtained in the present investigation demonstrate the range of conditions, where the BA transition can be characterized without perturbations by condensation or aggregation. The claim (Hormeno et al. 2011) that "condensation prevails over B-A transition in the structure of DNA at low humidity" is correct for the conditions of their experiments but is not correct in general. The experimental data supporting this claim were obtained at a too high salt concentration. It is well known that the tendency for condensation and aggregation is reduced at low-salt concentrations. Furthermore, the absence of a "contour length 
change compatible with a cooperative transition between the A and B form" is characterized here by measurements of the chain length dependence as a special effect in the range of high chain lengths used by Hormeno et al.: changes of the contour length are hidden in the broad distribution of wormlike chain configurations. Previously published experiments on a 70 bp poly[d(AT)] sample (Jose and Porschke 2004) demonstrated already the expected length reduction upon the BA transition without perturbation by condensation or aggregation. However, a detailed analysis of the various factors over a broad range of different chain lengths had not been presented yet. As described independently in a recent publication (Vafabakhsh and Ha 2012), the singlemolecule approach by force extension experiments can be applied only in a limited range. Thus, electro-optical techniques continue to be very useful for the analysis of DNA structures in solution. Electro-optics is particularly powerful in the range of low-salt concentrations where processes like the BA transition of DNA can be analyzed without perturbations.

The results of the present investigation were obtained over a range of different salt concentrations, but the analysis was restricted to buffers with $\mathrm{Na}^{+}$cations. Some results reported in the literature demonstrate that the nature of the cation can have a significant effect (Vorlickova et al. 1982). A clear influence of a detail in the solvent composition is demonstrated here by the comparison of ethanol with trifluoroethanol for reduction of the water activity. Thus, details of the solvent composition should be considered in more detail.

For future investigations, it may be of interest that intermolecular aggregation and precipitation are relatively slow processes at the low-DNA concentrations, which are sufficient for various spectroscopic types of analysis. Thus, there is a time window available for studies of A-DNA even in the critical range of conditions, where aggregation and precipitation cannot be avoided at long times.

Acknowledgments Open access funding provided by Max Planck Society. The facilities of the Gesellschaft für wissenschaftliche Datenverarbeitung, Göttingen, were used for part of data evaluations and computations.

\section{Compliance with ethical standards}

Conflict of interest The author declares that there are no conflicts of interest.

Open Access This article is distributed under the terms of the Creative Commons Attribution 4.0 International License (http://creativecommons.org/licenses/by/4.0/), which permits unrestricted use, distribution, and reproduction in any medium, provided you give appropriate credit to the original author(s) and the source, provide a link to the Creative Commons license, and indicate if changes were made.

\section{References}

Arnott S, Selsing E (1975) The conformation of C-DNA. J Mol Biol 98:265-269

Bloomfield VA (1996) DNA condensation. Curr Opin Struct Biol 6:334-341

Bloomfield VA, Crothers DM, Tinoco IJ (2000) Nucleic acidsstructures, properties and functions. University Science Books, Sausalito

Bokma JT, Johnson WC, Blok J (1987) CD of the Li-salt of DNA in ethanol/water mixtures-evidence for the B- to C-form transition in solution. Biopolymers 26:893-909

Brahms J, Mommaerts WFH (1964) Study of conformation of nucleic acids in solution by means of circular dichroism. J Mol Biol 10:73-88

Brahms J, Pilet J, Lan TTP, Hill LR (1973) Direct evidence of C-like form of sodium deoxyribonucleate. Proc Natl Acad Sci USA 70:3352-3355

Charney E, Chen HH (1987) Structure of A-DNA in solution. Proc Natl Acad Sci USA 84:1546-1549

Cheetham GMT, Steitz TA (1999) Structure of a transcribing T7 RNA polymerase initiation complex. Science 286:2305-2309

Diekmann S, Hillen W, Morgeneyer B, Wells RD, Porschke D (1982) Orientation relaxation of DNA restriction fragments and the internal mobility of the double helix. Biophys Chem 15:263-270

Diekmann S, Jung M, Teubner M (1984) On the orientation function of the electric dichroism of DNA. J Chem Phys 80:1259-1262

DiMaio F, Yu X, Rensen E, Krupovic M, Prangishvili D, Egelman EH (2015) A virus that infects a hyperthermophile encapsidates A-form DNA. Science 348:914-917

Esteve X, Patil KR, Fernandez J, Coronas A (1995) Prediction of density and excess volume for the ternary mixture-(water plus 2,2,2-trifluoroethanol plus 2,5,8,11,14-pentaoxapentadecane) from experimental binary values at temperatures from 283.15 to 333.15 K. J Chem Thermodyn 27:281-292

Franklin RE, Gosling RG (1953) The structure of sodium thymonucleate fibres. 1. The influence of water content. Acta Crystallogr 6:673-677

Fredericq E, Houssier C (1973) Electric dichroism and electric birefringence. Clarendon Press, Oxford

Girod JC, Johnson WC, Huntington SK, Maestre MF (1973) Conformation of deoxyribonucleic acid in alcohol solutions. Biochemistry 12:5092-5096

Grünhagen HH (1974) Entwicklung einer E-Feldsprungapparatur mit optischer Detektion und ihre Anwendung auf die Assoziation amphiphiler Elektrolyte. University of Braunschweig, Germany

Hagerman PJ, Zimm BH (1981) Monte-Carlo approach to the analysis of the rotational diffusion of wormlike chains. Biopolymers 20:1481-1502

Herbeck R, Yu TJ, Peticolas WL (1976) Effect of cross-linking on secondary structure of DNA.1. Cross-linking by photodimerization. Biochemistry 15:2656-2660

Hillen W, Wells RD (1980) Circular-dichroism studies of the B $\rightarrow$ A conformational transition in seven small DNA restriction fragments containing the Escherichia coli lactose control region. Nucleic Acids Res 8:5427-5444

Hormeno S, Moreno-Herrero F, Ibarra B, Carrascosa JL, Valpuesta JM, Arias-Gonzalez JR (2011) Condensation prevails over B-A transition in the structure of DNA at low humidity. Biophys $\mathrm{J}$ 100:2006-2015

Ivanov VI, Krylov DY (1992) A-DNA in solution as studied by diverse approaches. Method Enzymol 211:111-127

Ivanov VI, Minchenkova LE, Schyolkina AK, Poletayev AI (1973) Different conformations of double-stranded nucleic-acid in solution as revealed by circular-dichroism. Biopolymers 12:89-110 
Ivanov VI, Minchenkova LE, Minyat EE, Frank-Kamenetski MD, Schyolkina AK (1974) The B to A transition of DNA in solution. J Mol Biol 87:817-833

Jacobo-Molina A, Ding JP, Nanni RG, Clark AD, Lu XD, Tantillo C, Williams RL, Kamer G, Ferris AL, Clark P, Hizi A, Hughes SH, Arnold E (1993) Crystal-structure of human-immunodeficiencyvirus type-1 reverse-transcriptase complexed with doublestranded DNA at 3.0 angstrom resolution shows bent DNA. Proc Natl Acad Sci USA 90:6320-6324

Jones S, van Heyningen P, Berman HM, Thornton JM (1999) ProteinDNA interactions: a structural analysis. J Mol Biol 287:877-896

Jose D, Porschke D (2004) Dynamics of the B-A transition of DNA double helices. Nucleic Acids Res 32:2251-2258

Kiefer JR, Mao C, Braman JC, Beese LS (1998) Visualizing DNA replication in a catalytically active Bacillus DNA polymerase crystal. Nature 391:304-307

Knee KM, Dixit SB, Aitken CE, Ponomarev S, Beveridge DL, Mukerji I (2008) Spectroscopic and molecular dynamics evidence for a sequential mechanism for the A-to-B transition in DNA. Biophys J 95:257-272

Lang D (1969) Collapse of single DNA molecules in ethanol. J Mol Biol 46:209

Lu XJ, Shakked Z, Olson WK (2000) A-form conformational motifs in ligand-bound DNA structures. J Mol Biol 300:819-840

Nishimura Y, Torigoe C, Tsuboi M (1985) An A-Form Poly(dG). Poly $(\mathrm{dC})$ in $\mathrm{H}_{2} \mathrm{O}$ Solution. Biopolymers 24:1841-1844

Olive F, Chaudhari SK, Patil KR, Coronas A (1996) Viscosity of the binary systems containing trifluoroethanol, water and tetraethylene glycol dimethyl ether. Prediction of the ternary viscosity from binary data. Can J Chem Eng 74:163-169

Porschke D (1980) Structure and dynamics of a tryptophanepeptidepolynucleotide complex. Nucleic Acids Res 8:1591-1612

Porschke D (1984) Dynamics of DNA condensation. Biochemistry 23:4821-4828

Porschke D (1986) Structure and dynamics of double helices in solution-modes of DNA bending. J Biomol Struct Dyn 4:373-389

Porschke D (1991a) Nature of protamine DNA complexes-a special type of ligand-binding cooperativity. J Mol Biol 222:423-433

Porschke D (1991b) Persistence length and bending dynamics of DNA from electrooptical measurements at high salt concentrations. Biophys Chem 40:169-179

Porschke D, Antosiewicz JM (2007) Quantitative molecular electrooptics: macromolecular structures and their dynamics in solution. In: Stoylov SP, Stoimenova MV (eds) Molecular and colloidal electro-optics. CRC, Boca Raton, pp 55-107
Porschke D, Jung M (1985) The conformation of single stranded oligonucleotides and of oligonucleotide-oligopeptide complexes from their rotation relaxation in the nanosecond time range. $\mathbf{J}$ Biomol Struct Dyn 2:1173-1184

Potaman VN, Bannikov YA, Shlyachtenko LS (1980) Sedimentation of DNA in ethanol-water solutions within the interval of B-A transition. Nucleic Acids Res 8:635-642

Saenger W (1984) Principles of nucleic acid structure. Springer, New York

Shapiro DJ (1981) Quantitative ethanol precipitation of nanogram quantities of DNA and RNA. Anal Biochem 110:229-231

Tirado MM, Garcia de la Torre J (1980) Rotational-dynamics of rigid, symmetric top macromolecules-application to circular-cylinders. J Chem Phys 73:1986-1993

Tisne C, Hantz E, Hartmann B, Delepierre M (1998) Solution structure of a non-palindromic 16 base-pair DNA related to the HIV-1 kappa B site: evidence for BI-BII equilibrium inducing a global dynamic curvature of the duplex. J Mol Biol 279:127-142

Trifonov EN, Tan RKZ, Harvey SC (1987) Static persistence length of DNA. In: Olson WK, Sarma MH, Sarma RH, Sundaralingam M (eds) Structure and expression, vol 3: DNA bending and curvature. Adenine Press, Schenectady, pp 243-253

Vafabakhsh R, Ha T (2012) Extreme bendability of DNA less than 100 base pairs long revealed by single-molecule cyclization. Science 337:1097-1101

van Dam L, Levitt MH (2000) BII nucleotides in the B and C forms of natural-sequence polymeric DNA: a new model for the $\mathrm{C}$ form of DNA. J Mol Biol 304:541-560

Vorlickova M, Sedlacek P, Kypr J, Sponar J (1982) Conformational transitions of poly(dA-dT)-Poly(dA-dT) in ethanolic solutions. Nucleic Acids Res 10:6969-6979

Wu HM, Dattagupta N, Crothers DM (1981) Solution structural studies of the A-form and Z-form of DNA. Proc Natl Acad Sci Biol 78:6808-6811

Zavriev SK, Minchenkova LE, Frank-Kamenetskii MD, Ivanov VI (1978) Flexibility of boundaries between A-form and B-form sections in DNA molecule. Nucleic Acids Res 5:2657-2663

Zimmerman SB, Pheiffer BH (1979) Direct demonstration that the ethanol-induced transition of DNA is between the A-forms and B-forms - X-ray-diffraction study. J Mol Biol 135:1023-1027

Zimmerman SB, Pheiffer BH (1980) Does DNA adopt the C-form in concentrated salt-solutions or in organic-solvent water mixtures-an X-ray-diffraction study of DNA fibers immersed in various media. J Mol Biol 142:315-330 